\title{
Frugivoria por aves em Nectandra megapotamica (Lauraceae) em uma área de Floresta Estacional Decidual no Rio Grande do Sul, Brasil
}

\author{
Marilise M. Krügel ${ }^{1}$, Maria Inês Burger ${ }^{2} \&$ Marco A. Alves $^{3}$
}

1. UNIJUÍ, Departamento de Biologia e Química, Rodovia RS 344, km 39, 98900-000 Santa Rosa, RS. (marilisek@brturbo.com.br)

2. Rua Antônio da Silva Só, 432, 91780-170, Bairro Belém Novo, Porto Alegre, RS. (mines@hotmail.com)

3. Rua Ernesto Pereira, 673/201, 97105-140, Bairro Camobi, Santa Maria, RS. (marcoazambuja@yahoo.com.br)

\begin{abstract}
Bird frugivory on Nectandra megapotamica (Lauraceae) in an area of deciduous seasonal forest in Rio Grande do Sul, Brazil. The importance of Lauraceae fruits has been reported on the diet of several birds, mainly from the Ramphastidae, Cotingidae and Trogonidae families. The objectives of this study were to determine which birds consume the fruits of Nectandra megapotamica (Spreng.) Mez (Lauraceae) in a deciduous seasonal forest and, based on the analysis of some of the qualitative and quantitative dispersal components, infer which birds can act as dispersers of the seeds. The study was carried out at the Campo de Instrução de Santa Maria (CISM) (29 $43^{\prime}$ S, $53^{\circ} 42^{\prime} \mathrm{W}$ ), in the vicinity of Santa Maria, in Rio Grande do Sul State, Brazil. In a total of 70 hours of focal observation, 726 visits of 21 bird species were registered. The birds considered as potential dispersers of N. megapotamica were Turdus albicollis Vieillot, 1818, T. rufiventris Vieillot, 1818, Pitangus sulphuratus (Linnaeus, 1766) and T. amaurochalinus Cabanis, 1850 among the residents, and Tyrannus savanna Vieillot, 1808 and Myiodynastes maculatus (Müller, 1766) among the migratory ones. Generalist birds seemed to enhance the dispersal of $N$. megapotamica, since they consumed the entire fruits, realized short visits (shorter than 3 minutes) and presented high frequency of visits which, in turn, is related to a higher rate of fruit removal. Nectandra megapotamica shows features that allow it to be included in the generalist dispersal system, except for the high nutritional level of its seeds.
\end{abstract}

KEYWORDS. Nectandra megapotamica, Lauraceae, frugivory, birds, deciduous seasonal forest.

RESUMO. A importância dos frutos de Lauraceae tem sido relatada na dieta de muitas aves, principalmente das famílias Ramphastidae, Cotingidae e Trogonidae. Os objetivos deste estudo foram determinar quais espécies de aves consomem os frutos de Nectandra megapotamica (Spreng.) Mez (Lauraceae) numa área de Floresta Estacional Decidual e, com base na análise de alguns dos componentes qualitativos e quantitativos da dispersão, inferir quais aves podem atuar como dispersores de suas sementes. O estudo foi desenvolvido no Campo de Instrução de Santa Maria (CISM) (29 $43^{\prime} \mathrm{S}$, $\left.53^{\circ} 42^{\prime} \mathrm{W}\right)$, município de Santa Maria, Rio Grande do Sul. Num total de $70 \mathrm{~h}$ de observação focal, foram registradas 726 visitas de 21 espécies de aves. As aves consideradas como potencialmente dispersoras de $N$. megapotamica foram Turdus albicollis Vieillot, 1818, T. rufiventris Vieillot, 1818, Pitangus sulphuratus (Linnaeus, 1766) e T. amaurochalinus Cabanis, 1850 entre as residentes, e Tyrannus savanna Vieillot, 1808 e Myiodynastes maculatus (Müller, 1766) entre as migratórias. As aves com dieta generalista pareceram favorecer a dispersão de $N$. megapotamica, pois consumiram os frutos inteiros, realizaram visitas curtas (menos de 3 minutos) e apresentaram maior freqüência de visitação que, por sua vez, está relacionada a uma maior remoção dos frutos. Nectandra megapotamica possui características que a incluem no sistema de dispersão generalista, exceto pelo alto valor nutritivo dos seus frutos.

PALAVRAS-CHAVE. Nectandra megapotamica, Lauraceae, frugivoria, aves, Floresta Estacional Decidual.

Os animais frugívoros exercem um importante papel na demografia das comunidades vegetais pois: a) interagem no momento em que as plantas estão no estágio final do ciclo reprodutivo, podendo favorecer ou comprometer o sucesso desta fase (JORDANO, 1989); b) o padrão de deposição das sementes no ambiente pelos frugívoros afeta diretamente a sobrevivência das sementes e o estabelecimento das plântulas (Howe et al., 1985; Katusic-Malmborg \& Willson, 1988), e c) promovem a distribuição espacial dos futuros indivíduos adultos na floresta ao descartar as sementes após a ingestão (JANZEN et al., 1976; JoRDANO, 1992). Essas considerações são relevantes uma vez que, nas florestas tropicais, 50 a $90 \%$ das espécies arbóreas produzem frutos adaptados para a dispersão por animais (HowE \& SMALLWOOD, 1982).

Os estudos de frugivoria e/ou dispersão de sementes com aves permitem fazer inferências sobre a eficiência de uma determinada espécie como agente no processo de dispersão. Essa eficiência está relacionada tanto a componentes quantitativos (número de visitas e de frutos removidos) como qualitativos (qualidade do tratamento dado à semente no bico, o local e o padrão de deposição das sementes no ambiente) (Schupp, 1993), tendo conseqüências diretas no sucesso reprodutivo de uma planta (Howe et al., 1985; LoISELle \& BlAKE, 1999). Grande parte dos estudos envolvendo aves foram realizados com auto-ecologia de frugívoros (SNOw, 1970; Beehler, 1983; Wheelwright, 1983; Mikich, 2002) e observação de uma assembléia de aves utilizando os frutos de determinada espécie de planta (Howe \& Primack, 1975; McDiarmid et al., 1977; Fleming et al., 1985; Foster, 1990; Galetti \& Stotz, 1996; Pizo, 1997; Jordano \& Schupp, 2000; Francisco \& Galetti, 2001; CAZETTA et al., 2002).

A importância dos frutos de Lauraceae foi relatada na dieta de muitas aves, principalmente das famílias Ramphastidae, Cotingidae e Trogonidae (SKUTCH, 1944; Santana \& Milligan, 1984; WheElWright et al., 1984; Wheelwright, 1985; Ávila et al., 1996; Solórzano et al., 2000). No entanto, a família Lauraceae ainda é pouco estudada no Brasil. Francisco \& Galetti (2002) 
investigaram as aves como potenciais dispersoras de Ocotea pulchella Mart. numa área de vegetação de cerrado do sudeste brasileiro. Em um fragmento de Floresta Estacional Semidecidual do Paraná, MІкICH (2002) relatou alta freqüência no consumo de Nectandra megapotamica (Spreng.) Mez por Penelope superciliaris Temminck, 1815. No Parque Estadual Intervales (São Paulo), GALETti et al. (2000) registraram o consumo desta espécie por Ramphastidae. Na mesma área, $N$. megapotamica e outras onze espécies de lauráceas foram mencionadas na dieta de cotingídeos por Pizo et al. (2002).

Os objetivos deste estudo foram determinar quais espécies de aves consomem os frutos de $N$. megapotamica em uma área de Floresta Estacional Decidual no Rio Grande do Sul e inferir quais aves podem atuar como dispersores de suas sementes, avaliando diferenças entre elas quanto ao comportamento empregado na coleta e na ingestão dos frutos, número de frutos consumidos, freqüência de visitação e tempo de permanência sobre as plantas.

\section{MATERIAL E MÉTODOS}

Área de estudo. O estudo foi desenvolvido no Campo de Instrução de Santa Maria (CISM) (294'으. $\left.53^{\circ} 42^{\prime} \mathrm{W}\right)$, área pertencente ao Ministério da Defesa. Localiza-se no município de Santa Maria, Distrito de São Valentim, uma área de transição entre o Planalto Meridional Brasileiro e a Depressão Central do Rio Grande do Sul (Pereira et al., 1989). Possui 5.876 ha, dos quais cerca de $20 \%$ compreendem fragmentos florestais naturais de diferentes tamanhos, cercados por campos e várzeas. $\mathrm{O}$ fragmento estudado é o maior, com aproximadamente 1.000 ha. O relevo da área em questão é bastante heterogêneo, apresentando regiões com topografia levemente ondulada contrastando com outras com declividade acentuada (FARIAS et al.,1994).

A cobertura florestal da região de estudo pertence ao domínio da Floresta Estacional Decidual. Entre as espécies mais freqüentes nesta formação vegetal estão o angico-vermelho (Parapiptadenia rigida (Benth.) Brenan), a timbaúva (Enterolobium contortisiliquum (Vell.) Morong.), os camboatás (Cupania vernalis Cambess e Matayba elaeagnoides Radlk), as canelas (Ocotea spp. e Nectandra spp.), o açoita-cavalo (Luehea divaricata Mart.), o cincho (Sorocea bonplandii (Bail.) Burger, Lanj. \& Boer) e o catiguá (Trichilia clausseni C. DC.) (KLeIN, 1984; Leite \& Klein, 1990).

A região de estudo possui clima temperado chuvoso e quente do tipo Cfa (classificação climática de Köppen), com chuvas bem distribuídas ao longo do ano, temperatura média anual entre 18 a $20^{\circ} \mathrm{C}$ e precipitação média anual variando de 1.500 a $1.750 \mathrm{~mm}$ (Pereira et al., 1989).

Espécie estudada. O gênero Nectandra inclui aproximadamente 114 espécies com distribuição geográfica restrita à Região Neotropical (RoHwER \& KuBITZKI, 1993). No Brasil, N. megapotamica, conhecida popularmente por canela-preta, ocorre nos estados de São Paulo, Mato Grosso do Sul, Paraná, Santa Catarina e Rio Grande do Sul (Reitz et al., 1988; LoRENZI, 1992). Possui ampla distribuição geográfica no Rio Grande do Sul, integrando a composição florística da Floresta Ombrófila Densa, Floresta Ombrófila Mista e das Florestas Estacionais Decidual e Semidecidual (MARCHIORI, 1997).

Nectandra megapotamica destaca-se por ser uma das espécies mais freqüentes e características das florestas da Encosta Meridional da Serra Geral do Rio Grande do Sul (Machado \& Longhi, 1990; Farias et al., 1994). É uma espécie perenifólia, de grande porte (até 30 $\mathrm{m}$ de altura) e tronco com até $80 \mathrm{~cm}$ de diâmetro. Os frutos são ovóides e de coloração castanho-escura, ocorrendo a maturação no verão (MARCHIORI, 1997). O consumo dos frutos por aves é mencionado por LoRENZI (1992) mas inexistem estudos específicos de frugivoria.

Métodos de amostragem. Foram realizadas observações focais em sete indivíduos de $N$. megapotamica (10 h em cada planta), nos meses de dezembro de 2001, janeiro, fevereiro e dezembro de 2002 e janeiro de 2003. O maior esforço amostral ocorreu em janeiro, $27 \mathrm{~h}$ em 2002 e $30 \mathrm{~h}$ em 2003. Em dezembro e fevereiro, que correspondem ao início e término da maturação dos frutos, respectivamente, foram realizadas $13 \mathrm{~h}$ de observação. Os indivíduos foram selecionados ao longo de trilhas no interior da floresta. Os dados foram coletados em sessões de observação de 1 h, 2 h ou 3 h, realizadas entre 6:00 h e 11:00 h, com o auxílio de binóculo $8 \times 30$, permanecendo o observador distante cerca de 10 $\mathrm{m}$ da planta. Em cada sessão os seguintes dados foram registrados: espécies de aves visitantes, número de indivíduos de cada espécie, número de frutos consumidos, horário e duração da visita, comportamento empregado na coleta dos frutos (pousado ou em vôo), modo de mandibulação dos frutos e interações agonísticas intrae interespecíficas (perseguições em vôo, investidas diretas, mesmo sem haver contato físico) (Pızo, 1997; Galetti et al., 2003).

As visitas à planta foram classificadas como "completa", quando a ave pôde ser acompanhada desde a sua chegada à copa até a saída, ou "incompleta", quando somente parte da visita pôde ser acompanhada. Somente visitas completas foram utilizadas para calcular a média do tempo das visitas e do número de frutos consumidos. Definiu-se como visita toda vez que a ave chegava à árvore, independente de consumir ou não seus frutos. Quando a ave apanhava os frutos em vôo, sem pousar na planta, considerou-se o tempo de duração da visita como sendo de um segundo.

Quando um grupo de indivíduos de uma mesma espécie estava forrageando ao mesmo tempo na árvore, o número de frutos consumidos e o tempo de permanência foi registrado para um dos indivíduos, escolhido ao acaso.

Quanto ao modo de mandibulação, as aves foram agrupadas em três categorias: a) aquelas que engoliram os frutos inteiros, b) as que mandibularam, consumindo partes da polpa, e c) aquelas que quebraram e maceraram os frutos, consideradas predadoras de sementes (LEVEY, 1987; Pizo, 1997).

A porcentagem relativa de consumo para cada espécie de ave foi calculada dividindo o número total de frutos consumidos pela espécie $i$ pela soma dos frutos consumidos por todas as espécies e multiplicando-se o 
valor obtido por 100. Para o cálculo da freqüência de visitação (FV) de cada espécie, as sessões de observação foram padronizadas em $30 \mathrm{~min}$.

$\mathrm{FV}=\mathrm{n} / \mathrm{N}$, onde $\mathrm{n}=$ número de sessões em que determinada espécie esteve presente e $\mathrm{N}=$ número total de sessões.

A massa corporal das aves e a largura do bico (ao nível da extremidade das comissuras) foram obtidas a partir de exemplares disponíveis nas coleções do Museu de Ciências Naturais da Fundação Zoobotânica do Rio Grande do Sul e do Museu de Ciências e Tecnologia da Pontifícia Universidade Católica do Rio Grande do Sul ( $\mathrm{n}=1$ a 6 indivíduos por espécie). Na ausência de exemplares nessas instituições, foram utilizados dados da literatura. A ordem sistemática das aves seguiu BENCKE (2001). A classificação quanto ao status migratório foi baseado em Sick (1997), BENCKE (2001) e observações em campo.

Em cinco indivíduos de $N$. megapotamica foram coletados vinte frutos maduros de cada um, para obtenção das medidas de comprimento, largura (paquímetro digital, precisão de $0,1 \mathrm{~mm}$ ) e peso (balança eletrônica, precisão de $0,001 \mathrm{~g}$ ). Amostras da polpa foram enviadas para o Laboratório de Nutrição Animal da Universidade Federal do Rio Grande do Sul, Porto Alegre, para análise do conteúdo nutricional.

A relação entre massa corporal e consumo foi avaliada para cada espécie pela aplicação do coeficiente de correlação de Spearman aos valores médios da massa das aves e do número de frutos consumidos. As análises de diferenças no consumo de frutos e tempo de permanência na planta, entre as espécies, foram realizadas pela aplicação do teste de Kruskal-Wallis e ANOVA(Teste de Duncan). As aves com menos de quatro visitas completas não foram incluídas nessas análises. O programa SAS, versão 6.0 (SAS, 1989), foi utilizado para as análises.

\section{RESULTADOS}

Frutos maduros de N. megapotamica foram encontrados na área de estudo nos meses de dezembro, janeiro e início de fevereiro, sendo o pico da frutificação em janeiro. Os frutos mediram (média \pm desvio padrão) 9,64 $\pm 1,20 \mathrm{~mm}$ de comprimento, 6,89 $\pm 0,70 \mathrm{~mm}$ de largura e pesaram 0,33 $\pm 0,08 \mathrm{~g}$. Nectandra megapotamica apresenta uma semente cujo comprimento médio é de $9,11 \pm 1,09 \mathrm{~mm}$, largura de 5,65 $\pm 0,62 \mathrm{~mm}$ e peso de $0,15 \pm 0,05 \mathrm{~g}$. A relação do valor médio do fruto e da semente mostra que os frutos contêm aproximadamente $52,7 \%$ de polpa. A análise do conteúdo nutricional revelou que a matéria seca dos frutos apresenta 58,94\% de lipídios, $12,78 \%$ de cinzas, $11,01 \%$ de proteína bruta, $9,09 \%$ de extrato não nitrogenado e $8,18 \%$ de fibra bruta. A quantidade de água é de $56,16 \%$.

Vinte e uma espécies de aves consumiram os frutos de $N$. megapotamica nas $70 \mathrm{~h}$ de observações, num total de 726 visitas (Tab. I). Essas 21 espécies representam $46,7 \%$ do total de espécies de aves registradas consumindo frutos de diversas espécies de plantas no CISM (M.M.K., obs. pessoal). Entre as famílias registradas, Tyrannidae foi a melhor representada, com 10 espécies. Nela estão incluídas nove das dez espécies migratórias que utilizaram os frutos desta planta (marcadas com asterisco na Tab. I). A observação de um representante do gênero Elaenia, pela dificuldade de identificação envolvida, pode ser atribuída a qualquer uma das três espécies do gênero registradas na área de estudo: Elaenia parvirostris Pelzeln, 1868, E. spectabilis Pelzeln, 1868 e E. mesoleuca (Deppe, 1830). Além das aves, N. megapotamica foi consumida pelo bugio-ruivo (Alouatta guariba Cabrera, 1940) (Atelidae).

A curva cumulativa de espécies atingiu uma assíntota a partir de $30 \mathrm{~h}$ de observação. Três espécies foram acrescidas após este período [Elaenia sp., Chiroxiphia caudata (Shaw, 1793) e Saltator similis d'Orbigny \& Lafresnaye, 1837]. No entanto, essas espécies tiveram uma inexpressiva participação nas visitas e na remoção dos frutos (Tab. I). Sendo assim, o total de horas empregado parece ter sido suficiente para amostrar as principais espécies de aves que consomem os frutos de N. megapotamica na área de estudo (Fig. 1).

O tamanho das aves que consomem $N$. megapotamica variou desde espécies pequenas (16 g para Vireo olivaceus (Linnaeus, 1766)) até espécies de grande porte como Penelope obscura Temminck, 1815, com mais de $1 \mathrm{~kg}$. Todas as aves têm a largura do bico maior que a média da largura dos frutos (Tab. I).

As espécies de aves residentes no CISM foram responsáveis por $64,6 \%$ das visitas e $75,7 \%$ dos frutos consumidos, enquanto as migratórias somaram $35,4 \%$ das visitas e $24,3 \%$ do consumo. Com relação ao consumo, seis espécies, Turdus albicollis Vieillot, 1818, T. rufiventris Vieillot, 1818, Myiodynastes maculatus (Müller, 1776), Tyrannus savanna Vieillot, 1808, Turdus amaurochalinus Cabanis, 1850 e Pitangus sulphuratus (Linnaeus, 1766) somaram $84,3 \%$ das visitas à planta, destacando-se $T$. albicollis, com quase $30 \%$. Por outro lado, Elaenia sp., C. caudata e $S$. similis realizaram somente uma visita. As maiores freqüências de visitação também foram registradas para poucas espécies, a saber: T. albicollis, T. rufiventris, M. maculatus e T. amaurochalinus. As maiores porcentagens de consumo de frutos foram obtidas para $T$. albicollis $(34,6 \%)$ seguida por $T$. rufiventris $(17,4 \%), T$. amaurochalinus $(11 \%), P$.

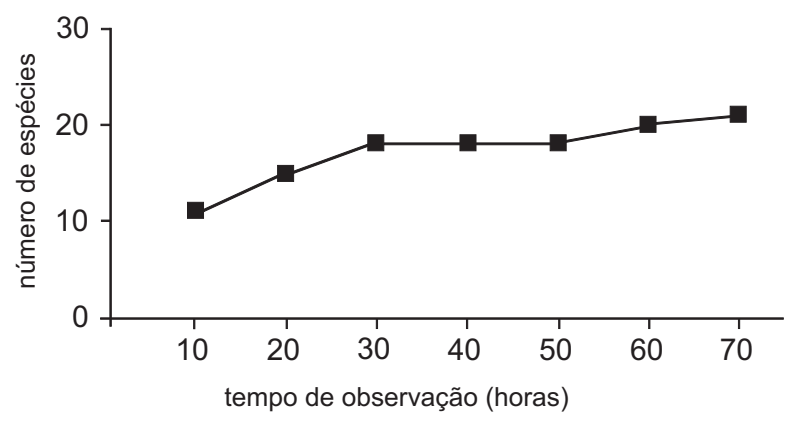

Fig. 1. Curva cumulativa da riqueza de espécies de aves que consumiram os frutos de Nectandra megapotamica em relação ao tempo de observação. 
Tabela I. Relação das espécies observadas consumindo os frutos de Nectandra megapotamica em uma área de Floresta Estacional Decidual, município de Santa Maria, RS (*, espécies migratórias; **, não foi possível calcular o percentual pela ausência de visitas completas; ${ }^{1}$, número de visitas completas quanto ao tempo de permanência na árvore e quanto ao número de frutos consumidos; ${ }^{2}$, média e desvio padrão do número de frutos consumidos por visita completa; ${ }^{3}$, média e desvio padrão do tempo de permanência na árvore por visita completa; ${ }^{4}$, média e desvio padrão da largura do bico; ${ }^{5}, \mathrm{FV}=$ freqüência de visitação).

\begin{tabular}{|c|c|c|c|c|c|c|c|c|}
\hline $\begin{array}{l}\text { Família } \\
\text { Espécies }\end{array}$ & $\begin{array}{l}\mathrm{N}^{\circ} \text { total de } \\
\text { visitas }(\%)\end{array}$ & $\begin{array}{c}\text { Visitas } \\
\text { completas }^{1}\end{array}$ & Consumo $^{2}$ & $\begin{array}{l}\text { Tempo das } \\
\text { visitas }(\mathrm{s})^{3}\end{array}$ & $\begin{array}{l}\text { Largura do } \\
\text { bico }^{4}\end{array}$ & $\mathrm{FV}^{5}$ & $\begin{array}{l}\text { Percentual } \\
\text { de consumo }\end{array}$ & $\begin{array}{c}\text { Número de frutos } \\
\text { derrubados }(\%)\end{array}$ \\
\hline \multicolumn{9}{|l|}{ CRACIDAE } \\
\hline $\begin{array}{l}\text { Penelope obscura } \\
\text { Temminck, } 1815\end{array}$ & $5(0,7)$ & - & - & - & 24,3 & 0,04 & - & $3 * *$ \\
\hline \multicolumn{9}{|l|}{ PSITTACIDAE } \\
\hline $\begin{array}{l}\text { Aratinga leucophthalmus } \\
\text { (Müller, 1776) }\end{array}$ & $2(0,3)$ & - & - & - & - & 0,01 & - & - \\
\hline $\begin{array}{l}\text { Pyrrhura frontalis } \\
\text { (Vieillot, 1818) }\end{array}$ & $11(1,5)$ & - & - & - & $11,53 \pm 0,29$ & 0,07 & - & - \\
\hline \multicolumn{9}{|l|}{ TROGONIDAE } \\
\hline $\begin{array}{l}\text { Trogon surrucura } \\
\text { Vieillot, } 1817\end{array}$ & $4(0,6)$ & 1 & 2 & 180 & $18,61 \pm 1,28$ & 0,02 & 0,47 & - \\
\hline \multicolumn{9}{|l|}{ TYRANNIDAE } \\
\hline Elaenia sp.* & $1(0,1)$ & 1 & 1 & 25 & - & 0,007 & 0,06 & - \\
\hline $\begin{array}{l}\text { Myiarchus swainsoni } \\
\text { Cabanis \& Heine, 1859* }\end{array}$ & $15(2,1)$ & 7 & $1,3 \pm 0,5$ & $62,3 \pm 48,9$ & $10,27 \pm 0,54$ & 0,09 & 1,16 & $1(5,1)$ \\
\hline $\begin{array}{l}\text { Pitangus sulphuratus } \\
\quad \text { (Linnaeus, 1766) }\end{array}$ & $60(8,3)$ & 14 & $3,0 \pm 1,8$ & $97,7 \pm 89,2$ & $13,62 \pm 0,96$ & 0,18 & 10,7 & $1(0,5)$ \\
\hline $\begin{array}{l}\text { Megarynchus pitangua } \\
\text { (Linnaeus, 1766)* }\end{array}$ & $3(0,4)$ & 2 & $1 / 1$ & $38 / 1$ & 16,2 & 0,02 & 0,18 & - \\
\hline $\begin{array}{l}\text { Myiodynastes maculatus } \\
\text { (Müller, 1776)* }\end{array}$ & $98(13,5)$ & 43 & $1,6 \pm 1,1$ & $88,1 \pm 85,2$ & $14,01 \pm 0,38$ & 0,37 & 9,32 & $4(2,5)$ \\
\hline $\begin{array}{l}\text { Empidonomus varius } \\
\text { (Vieillot, 1818)* }\end{array}$ & $27(3,7)$ & 15 & $1,5 \pm 1,1$ & $117,1 \pm 97,5$ & $9,75 \pm 0,58$ & 0,14 & 2,41 & $2(4,9)$ \\
\hline $\begin{array}{l}\text { Tyrannus melancholicus } \\
\text { Vieillot, } 1819^{*}\end{array}$ & $21(2,9)$ & 5 & $2,2 \pm 1,8$ & $50,8 \pm 43,5$ & $12,8 \pm 0,60$ & 0,08 & 2,75 & $1(2,1)$ \\
\hline $\begin{array}{l}\text { Tyrannus savanna } \\
\text { Vieillot, } 1808^{*}\end{array}$ & $78(10,7)$ & 32 & $1,6 \pm 1,3$ & $28,5 \pm 42,6$ & $10,62 \pm 0,02$ & 0,12 & 7,42 & $3(2,4)$ \\
\hline $\begin{array}{l}\text { Pachyramphus polychopterus } \\
\text { (Vieillot, 1818)* }\end{array}$ & $4(0,6)$ & 1 & 1 & 65 & 10,97 & 0,03 & 0,24 & - \\
\hline $\begin{array}{l}\text { Tityra cayana } \\
\quad(\text { Linnaeus, } 1766)^{*}\end{array}$ & $3(0,4)$ & 1 & 2 & 80 & $14,73 \pm 1,74$ & 0,01 & 0,35 & $1(16,6)$ \\
\hline \multicolumn{9}{|l|}{ PIPRIDAE } \\
\hline $\begin{array}{l}\text { Chiroxiphia caudata } \\
\quad \text { (Shaw, 1793) }\end{array}$ & $1(0,1)$ & 1 & 1 & 10 & $9,31 \pm 0,55$ & 0,007 & 0,06 & - \\
\hline \multicolumn{9}{|l|}{ MUSCICAPIDAE } \\
\hline $\begin{array}{l}\text { Turdus rufiventris } \\
\quad \text { Vieillot, } 1818\end{array}$ & $98(13,5)$ & 39 & $3,0 \pm 2,1$ & $96,8 \pm 101,5$ & $11,60 \pm 0,47$ & 0,41 & 17,48 & $8(2,7)$ \\
\hline $\begin{array}{l}\text { Turdus amaurochalinus } \\
\quad \text { Cabanis, } 1850\end{array}$ & $62(8,5)$ & 17 & $3,0 \pm 2,8$ & $146,1 \pm 140,1$ & $10,56 \pm 0,97$ & 0,26 & 11,06 & $3(1,6)$ \\
\hline $\begin{array}{l}\text { Turdus albicollis } \\
\quad \text { Vieillot, } 1818\end{array}$ & $216(29,7)$ & 57 & $2,7 \pm 1,9$ & $105,7 \pm 145,9$ & $11,12 \pm 0,64$ & 0,67 & 34,68 & $15(2,6)$ \\
\hline \multicolumn{9}{|l|}{ EMBERIZIDAE } \\
\hline $\begin{array}{l}\text { Saltator similis } \\
\text { d'Orbigny \& Lafresnaye, } 1\end{array}$ & 337 & 1 & 1 & 104 & $11,20 \pm 0,59$ & 0,007 & 0,06 & - \\
\hline $\begin{array}{l}\text { Tangara preciosa } \\
\quad(\text { Cabanis, 1850) }\end{array}$ & $9(1,2)$ & 5 & $2,2 \pm 0,8$ & $73,6 \pm 44,7$ & $8,13 \pm 0,12$ & 0,05 & 1,18 & $4(20,2)$ \\
\hline \multicolumn{9}{|l|}{ VIREONIDAE } \\
\hline $\begin{array}{l}\text { Vireo olivaceus } \\
\qquad(\text { Linnaeus, 1766)* }\end{array}$ & $7(1,0)$ & 5 & 1 & $22,2 \pm 11,3$ & $8,29 \pm 0,37$ & 0,05 & 0,42 & $2(28,6)$ \\
\hline
\end{tabular}

sulphuratus $(10,7 \%)$ e M. maculatus $(9,3 \%)$. As demais espécies foram responsáveis por $17 \%$ do consumo (Tab. I).

As espécies de aves diferiram tanto no número de frutos consumidos por visita $(\mathrm{H}=43,17 ; \mathrm{p}=0,0001)$ quanto no tempo de duração da visita $(H=37,94 ; p=0,0001)$. A ANOVA (Teste de Duncan) mostrou diferença significativa com relação à permanência na árvore para $T$. amaurochalinus quando comparado com T. savanna e $V$. olivaceus. Com relação ao consumo, o grupo formado por T. rufiventris, $P$. sulphuratus e T. amaurochalinus diferiu de Myiarchus swainsoni Cabanis \& Heine, 1859 e $V$. olivaceus. A correlação foi significativa entre a massa das diferentes espécies e o número médio de frutos consumidos $\left(r_{s}=0,87 ; p=0,0004\right)$.

A duração da visita e o número de frutos 
consumidos foi positivamente correlacionado para $M$. maculatus $\left(\mathrm{r}_{\mathrm{s}}=0,43 ; \mathrm{p}=0,003\right)$, P. sulphuratus $\left(\mathrm{r}_{\mathrm{s}}=0,56\right.$; $\mathrm{p}=0,036), T$. albicollis $\left(\mathrm{r}_{\mathrm{s}}=0,61 ; \mathrm{p}=0,0001\right), T$. amaurochalinus $\left(\mathrm{r}_{\mathrm{s}}=0,76 ; \mathrm{p}=0,0004\right)$, T. rufiventris $\left(\mathrm{r}_{\mathrm{s}}=\right.$ $0,82 ; \mathrm{p}=0,0001)$ e $T$. savanna $\left(\mathrm{r}_{\mathrm{s}}=0,75 ; \mathrm{p}=0,0001\right)$. Não foi possível fazer o teste de correlação para $V$. olivaceus em razão do pequeno número de dados. Para as demais espécies, não foram encontradas correlações.

Dezessete espécies de aves engoliram os frutos inteiros. Três foram consideradas predadoras, Pyrrhura frontalis (Vieillot, 1818), Aratinga leucophthalmus (Müller, 1776) e $S$. similis, por triturar e danificar as sementes durante a ingestão. Somente Tangara preciosa (Cabanis, 1850) exibiu o comportamento de mandibular, o que provocou a queda do fruto em três das nove visitas à planta.

Empidonomus varius (Vieillot, 1818) $(\mathrm{n}=1), T$. albicollis $(\mathrm{n}=4)$, T. amaurochalinus $(\mathrm{n}=1)$ e T. rufiventris $(\mathrm{n}=1)$ foram observados regurgitando sementes intactas sob a planta-mãe. Treze espécies deixaram cair 2,8\% do total de frutos consumidos sob a planta-mãe após a remoção (Tab. I). Em termos percentuais, T. preciosa e $V$. olivaceus derrubaram acima de $20 \%$ dos frutos que removeram (Tab. I). O comportamento de carregá-los no bico ao deixar a planta foi observado para Trogon surrucura Vieillot, $1817(\mathrm{n}=1), E$. varius $(\mathrm{n}=1), T$. rufiventris $(\mathrm{n}=1), T$. albicollis adulto $(\mathrm{n}=1)$ e jovem $(\mathrm{n}$ $=1)$ e $V$. olivaceus $(\mathrm{n}=2)$. Além dessas, em uma oportunidade, Tyrannus melancholicus Vieillot, 1819 foi visto carregando dois frutos no bico, provavelmente para alimentar os filhotes.

Em 3,3\% das visitas, alguns indivíduos permaneceram na árvore por vários minutos após consumir os frutos. Em uma das sessões de observação, E. varius permaneceu parado por mais de 20 minutos. Outras espécies que exibiram este comportamento foram P. sulphuratus, M. swainsoni, M. maculatus, $T$. melancholicus, T. rufiventris, T. amaurochalinus e $T$. albicollis.

Os frutos foram consumidos tanto por espécies que coletam preferencialmente em vôo (como Tyrannidae e Trogonidae) quanto por aquelas que o realizam pousadas (Cracidae, Psittacidae, Emberizidae e Vireonidae). Muscicapidae e Pipridae empregam as duas formas. O fruto de $N$. megapotamica caracteriza-se por estar preso a uma cúpula, a qual proporciona certa dificuldade para arrancá-lo, especialmente quando em vôo. Foram registradas várias tentativas frustradas de coleta por E. varius, T. savanna, M. maculatus, $M$. swainsoni, jovem e adulto de T. albicollis e T.rufiventris, revelando por que muitos frutos observados no chão apresentaram o pericarpo com cortes. Tyrannus melancholicus e T. rufiventris apanharam o fruto junto com a cúpula algumas vezes, descartando-a em seguida, mas $P$. sulphuratus ingeriu tudo em uma ocasião.

Filhotes de $T$. preciosa e $T$. surrucura foram alimentados com frutos de $N$. megapotamica os quais foram engolidos inteiros após breve mandibulação. Jovens das espécies de Turdus foram responsáveis por $14,5 \%$ do total das visitas $(n=105)$.

Não foram registradas visitas completas de alimentação para $P$. obscura, $P$. frontalis e $A$. leucophthalmus. No entanto, $P$. obscura pôde ser acompanhada por uma hora e foi possível contar a ingestão de mais de 90 frutos até a ave esconder-se na copa.

A maioria das espécies chegava à planta sozinha. Psitacídeos chegavam em grupos de, no máximo, 10 indivíduos. Pitangus sulphuratus e T. savanna foram os únicos passeriformes que chegaram em grupos de três ou quatro indivíduos, possivelmente grupos familiares.

Os encontros agonísticos observados entre as aves visitantes não foram muito freqüentes $(\mathrm{n}=15)$. Turdus rufiventris e $M$. maculatus foram os principais agressores e, entre as mais agredidas, figuraram $T$. albicollis e $M$. maculatus. Cinco dos 15 encontros agonísticos foram intraespecíficos e ocorreram em P. sulphuratus, $M$. maculatus, E. varius e T. albicollis (Tab. II).

Tabela II. Interações agonísticas entre as espécies de aves que se alimentaram dos frutos de Nectandra megapotamica em uma área de Floresta Estacional Decidual, município de Santa Maria (Myiswa, Myiarchus swainsoni; Pitsul, Pitangus sulphuratus; Myimac, Myiodynastes maculatus; Empvar, Empidonomus varius; Tyrsav, Tyrannus savanna, Turruf, Turdus rufiventris; Turama, Turdus amaurochalinus; Turalb, Turdus albicollis).

\begin{tabular}{|c|c|c|c|c|c|c|}
\hline \multirow{2}{*}{$\begin{array}{l}\text { Espécies } \\
\text { agressoras }\end{array}$} & \multicolumn{6}{|c|}{ Espécies agredidas } \\
\hline & Tyrsav & Turalb & Turama & Myimac & Empvar & Pitsul \\
\hline Myiswa & 1 & & & & & \\
\hline Pitsul & & & & & & 1 \\
\hline Myimac & & 1 & 1 & 1 & & 1 \\
\hline Empvar & & & & & 1 & \\
\hline Turruf & & 3 & & 2 & & \\
\hline Turama & & & & 1 & & \\
\hline Turalb & & 2 & & & & \\
\hline
\end{tabular}

\section{DISCUSSÃO}

As espécies de Lauraceae possuem frutos com uma semente grande envolvida por um pericarpo fino e polpa com alto percentual de lipídios (SNOw, 1981). Na Costa Rica WHEELWRIGHT et al. (1984) observaram uma variação de 5,8 a 45,2\% de lipídeos em 15 espécies. A análise do conteúdo nutricional de $N$. megapotamica revelou altos percentuais de lipídios $(58,9 \%)$ e de proteína $(11,0 \%)$, comparável ao obtido nos frutos arilados de Meliaceae (Foster \& MacDiarmid, 1983) e Apocynaceae (MACDiarmid et al., 1977).

A maior parte das espécies que utilizaram os frutos de $N$. megapotamica no CISM são consideradas frugívoras oportunistas, isto é, têm uma dieta mista, composta por invertebrados e frutos. Outras, como $T$. surrucura e $C$. caudata, são citados como frugívoros especialistas, mas que podem complementar a dieta com matéria animal (SNOw, 1981; Sick, 1997). Quanto ao hábitat, as aves registradas são associadas a ambientes florestais e de área aberta.

A família Tyrannidae realizou $42,7 \%$ do total das visitas, sendo as espécies migratórias responsáveis por $34,4 \%$. Apesar de serem primariamente insetívoros (SicK, 1997), vários tiranídeos são conhecidos por incluir frutos em sua dieta, como o gênero Elaenia (MARINI \& 
Cavalcanti, 1998). O consumo de frutos por tiranídeos também foi registrado para espécies de Rapanea (Pineschi, 1990) e Trichilia (Gondim, 2001), Talauma ovata St. Hil. (CAZETTA et al., 2002), Ocotea pulchella (Nees et Mart. ex Nees) Ness (Francisco \& GaletTI, 2002) e Alchornea triplinervia (Spreng.) M. Arg. (KRÜGEL \& BEHR, 1999), entre outras.

Diferenças ecológicas e comportamentais entre os frugívoros podem afetar sua eficiência como dispersores (Snow, 1981; Levey, 1987; Loiselle \& Blake, 1999). Igualmente importantes são o tamanho corporal e a largura do bico (Pizo, 1996). Entre as espécies residentes, T. albicollis, T. rufiventris, T. amaurochalinus e $P$. sulphuratus são potenciais dispersoras por engolirem os frutos inteiros, apresentarem os maiores percentuais de consumo e de freqüência de visitação. Usando este critério, entre as aves migratórias, destacam-se $M$. maculatus e T. savanna como principais dispersores. As espécies predadoras de sementes somaram uma baixa freqüência nas visitas, presumivelmente não comprometendo a dispersão das sementes.

Migrantes neotropicais são importantes dispersores para algumas espécies vegetais (LECK, 1972; Howe \& De Steven, 1979; GreenberG, 1981). Em um estudo desenvolvido na Costa Rica, Blake \& Loiselle (1992) registraram frutos de 98 plantas em amostras fecais de 21 aves migratórias. Nectandra megapotamica frutifica numa época do ano em que a riqueza de espécies aumenta no Rio Grande do Sul com a chegada das aves migratórias (Belton, 1994), o que contribui para um aumento do número de dispersores. Nesse estudo, as espécies migratórias representam $47,6 \%$ dos consumidores, realizaram $35,4 \%$ das visitas e consumiram $24,3 \%$ dos frutos. No entanto, apenas M. maculatus e $T$. savanna tiveram maior participação nas visitas e no consumo de frutos.

A riqueza de aves utilizando os frutos de uma planta em região tropical pode ser grande, como registrado por KANTAK $(1979,1981)$ no México. Entretanto, o número de espécies potencialmente dispersoras pode ser bem menor, como evidenciado no CISM, onde 6 das 21 espécies reuniram características para tal. Das 27 espécies capturadas em um estudo sobre a dispersão de melastomatáceas na Estação Biológica La Selva (Costa Rica), LoISELlE \& BLAKE (1999) relataram que 95 a 99\% das sementes presentes nas fezes foram de apenas seis espécies de aves.

A largura do bico dos frugívoros é um fator limitante para o tamanho dos frutos que podem ser ingeridos inteiros (Moermond \& Denslow, 1985; WheELWright, 1985). Particularmente para N. megapotamica, este parece não ser um fator limitante, pois todas as aves apresentaram a largura do bico maior do que a largura do fruto. A maioria das aves engoliu os frutos inteiros, mas algumas, como $P$. polychopterus, $T$. preciosa e $V$. olivaceus, tiveram certa dificuldade em fazê-lo. Destas, T. preciosa foi a única que exibiu o comportamento de mandibular antes de ingerir o fruto inteiro, derrubando alguns em função do tamanho da semente. Para aves mandibuladoras, o tamanho da semente pode impedir que atuem como dispersores. LEVEY (1987) registrou um aumento na proporção de frutos derrubados durante a mandibulação em função do aumento do tamanho das sementes para vários traupíneos, constatando que as aves derrubaram a maioria das sementes maiores do que $4 \mathrm{~mm}$.

Snow \& Snow (1988) apud Jordano (1992) registraram um decréscimo no sucesso de mandibulação dos frutos quando a relação largura do fruto/largura do bico era maior do que 1 . De fato, as aves que tiveram o maior percentual de queda dos frutos removidos $(T$. preciosa e $V$. olivaceus) também foram aquelas que apresentaram os maiores valores para essa razão $(0,84 \mathrm{e}$ 0,83, respectivamente). Pizo (1997) relatou $V$. olivaceus como o principal consumidor de Cabralea canjerana (Vell.) Mart. (Meliaceae) na Mata de Santa Genebra (São Paulo) e também o responsável pela queda de $30 \%$ dos frutos removidos. $\mathrm{O}$ autor atribuiu este alto percentual à dificuldade de engolir os frutos em função da largura do bico e aos encontros agonísticos, em que esta foi a espécie mais agredida.

Sementes maiores são geralmente regurgitadas pelas aves (Pızo, 1996) e são descartadas mais rapidamente do que aquelas que passam por todo o trato digestório para serem defecadas (LeVEY, 1987). A permanência de algumas espécies na copa de $N$. megapotamica, possibilitando que os frutos removidos sejam regurgitados ou defecados sob a planta-mãe, pode ser considerada um dos pontos negativos na qualidade da dispersão. Mas, como ocorreu em apenas 3,3\% das visitas e o total de frutos derrubados somou $2,8 \%$, não parece comprometer o processo de dispersão.

Sementes de Ocotea pulchella foram encontradas nas fezes de Schistochlamys ruficapillus (Vieillot, 1817) (Emberizidae) em condição de cativeiro (FRANCISCO \& Galetti, 2002). O tamanho das sementes desta espécie e de $N$. megapotamica é semelhante, não podendo ser descartada a possibilidade de que também sejam eliminadas nas fezes.

Os encontros agonísticos ocorreram, principalmente, entre as espécies com maior freqüência de visitação. No entanto, foram pouco freqüentes, sugerindo não ser um fator prejudicial ao processo de dispersão. WiLLIS (1966) comentou que parece não haver competição e exclusão quando o alimento é abundante, sendo este o caso de $N$. megapotamica.

Foram constatados vários fatores que podem favorecer a dispersão de $N$. megapotamica, como a riqueza de espécies visitando a planta, as visitas curtas (menos de 3 minutos), a ingestão de frutos inteiros, a alta freqüência de visitação de algumas espécies que, por sua vez, está relacionada a uma maior remoção dos frutos.

McKEY (1975) propôs dois sistemas de dispersão relacionados aos tipos de frutos produzidos: o de especialistas, aqueles com sementes grandes, ricos em lipídios, produzidos em pequena quantidade e disponíveis por um longo período; e o de generalistas, no outro extremo, com sementes pequenas, ricos em carboidratos, produzidos em abundância e disponíveis por curto período. Nectandra megapotamica possui características que a incluem no sistema de dispersão generalista, exceto pelo alto valor nutritivo dos seus frutos. 
O presente estudo revelou que os frutos de $N$. megapotamica constituem um importante recurso alimentar para as aves. Esta espécie possui ampla distribuição no Rio Grande do Sul, frutifica durante o período reprodutivo das aves e seus frutos foram consumidos por muitas espécies de aves que podem proporcionar a deposição das sementes em diferentes sítios, uma vez que diferem quanto ao comportamento e uso do ambiente.

Agradecimentos. Somos gratos ao Ministério da Defesa pelo apoio e permissão de acesso à área de estudo. À Capes pelo apoio financeiro na forma de bolsa de doutorado. A Leandro Martins e Neander M. Heming pelo auxílio nas atividades de campo. A Magda S. Hentschke pela revisão gramatical. A Marco A. Pizo pelas sugestões que contribuíram para a realização deste estudo. Agradecemos a Ana Maria Rui, Everton R. Behr, Luiz dos Anjos, Nêmora P. Prestes e Solon J. Longhi e a dois revisores anônimos pelas valiosas sugestões para o aperfeiçoamento deste trabalho.

\section{REFERÊNCIAS BIBLIOGRÁFICAS}

Ávila, M. L.; Hernández, V. H. \& Velarde, E. 1996. The diet of Resplendent Quetzal (Pharomachrus mocinno mocinno: Trogonidae) in a Mexican Cloud Forest. Biotropica 28(4b):720-727.

BeEHLER, B. 1983. Frugivory and polygamy in birds of paradise. Auk 100:1-12.

Belton, W. 1994. Aves do Rio Grande do Sul: distribuição e biologia. São Leopoldo, Unisinos. 584p.

Bencke, G. A. 2001. Lista de referência das aves do Rio Grande do Sul. Porto Alegre, Fundação Zoobotânica do Rio Grande do Sul. 102p. (Publicações Avulsas FZB, 10).

Blake, J. G. \& Loiselle, B. A. 1992. Fruits in the diets of Neotropical migrant birds in Costa Rica. Biotropica 24(2):200-210.

Cazetta, E.; Rubim, P.; Lunardi, V. O.; Francisco, M. R. \& Galetti, M. 2002. Frugivoria e dispersão de sementes de Talauma ovata (Magnoliaceae) no sudeste brasileiro. Ararajuba 10(2): 199-206.

Farias, J. A. C.; Teixeira, I. F.; Pés, L. \& Alvarez Filho, A. 1994. Estrutura fitossociológica de uma Floresta Estacional Decidual na região de Santa Maria, RS. Ciência Florestal 4(1): 109-128.

Fleming, T. H.; Williams, C. F.; Bonaccorso, F. J. \& Herbst, L. H. 1985. Phenology, seed dispersal, and colonization in Mutingia calabura, a Neotropical pioneer tree. American Journal of Botany 72(3):383-391.

Foster, M. S. 1990. Factors influencing bird foraging preferences among conspecific fruit trees. Condor 92:844-854.

Foster, M. S. \& McDiarmid, R. W. 1983. Nutritional value of the aril of Trichilia cuneata, a bird-dispersed fruit. Biotropica 15:26-31.

Francisco, M. R. \& Galetti, M. 2001. Frugivoria e dispersão de sementes de Rapanea lancifolia (Myrsinaceae) por aves numa área de cerrado do Estado de São Paulo, sudeste do Brasil. Ararajuba 9(1):13-19.

2002. Aves como potenciais dispersoras de sementes de Ocotea pulchella Mart. (Lauraceae) numa área de vegetação de cerrado no sudeste brasileiro. Revista Brasileira de Botânica 25(1):11-17.

Galetti, M. \& Sтотz, D. 1996. Miconia hypoleuca (Melastomataceae) como espécie-chave para aves frugívoras no sudeste do Brasil. Revista Brasileira de Biologia 56(2):435-439.

Galetti, M. R.; Laps, R. \& Pizo, M. A. 2000. Frugivory by toucans (Ramphastidae) at two altitudes in the Atlantic forest of Brazil. Biotropica 32(4b):842-850.

Galetti, M.; Pizo, M. A. \& Morellato, P. L. 2003. Fenologia, frugivoria e dispersão de sementes. In: Cullen JR., L.; PÁduA, C. V. \& Rudran, R. org. Métodos de estudo em Biologia da
Conservação e Manejo da Vida Silvestre. Curitiba, Ed. da UFPR, Fundação O Boticário. p. 395-422.

Gondim, M. J. C. 2001. Dispersão de sementes de Trichilia spp. (Meliaceae) por aves em um fragmento de mata mesófila semidecídua, Rio Claro, SP, Brasil. Ararajuba 9(2):101-112.

Greenberg, R. 1981. Frugivory in some migrant tropical forest wood warbles. Biotropica 13:215-223.

Howe, H. F. \& Primack, R. B. 1975. Differential seed dispersal by birds of the tree Casearia nitida (Flacourtiaceae). Biotropica 7(4):278-283

Howe, H. F. \& De Steven, D. 1979. Fruit production, migrant bird visitation, and seed dispersal of Guarea glabra in Panama. Oecologia 39:185-196.

Howe, H. F. \& Smallwood, J. 1982. Ecology of seed dispersal. Annual Review of Ecology and Systematics 13:201-228.

Howe, H. F.; Schupp, E. W. \& Westley, L. C. 1985. Early consequences of seed dispersal for a neotropical tree (Virola surinamensis). Ecology 66:781-791.

Janzen, D. H.; Miller, G. A.; Hackforth-Jones, J.; Pond, C. M. Hooper, K. \& Janos, D. P. 1976. Two Costa Rican batgenerated seed shadows of Andira inermis (Leguminosae). Ecology 57:1068-1075.

Jordano, P. 1989. Pre-dispersal biology of Pistacia lentiscus (Anacardiaceae): cumulative effects on seed removal by birds. Oikos 55:375-386.

1992. Fruits and frugivory. In: FenNER, M. ed. Seeds: the ecology of regeneration in plant communities. Washington, C.A.B. International. p.105-156.

Jordano, P \& Schupp, E. W. 2000. Seed disperser effectiveness: the quantity component and patterns of seed rain for Prunus mahaleb. Ecological Monographs 70(4):591-615.

KANTAK, G. E. 1979. Observations on some fruit-eating birds in Mexico. Auk 96: 183-186.

. 1981. Temporal feeding patterns of some tropical frugivores. Condor 83:185-187.

Katusic-Malmborg, P. \& Willson, M. F. 1988. Foraging ecology of avian frugivores and some consequences for seed dispersal in an Illinois woodlot. Condor 90:173-186.

KLeIn, R. M. 1984. Síntese ecológica da Floresta Estacional da Bacia do rio Jacuí e importância do reflorestamento com essências nativas (RS). In: Congresso Florestal Estadual, $5^{\circ}$, Nova Prata. Anais... Porto Alegre, Secretaria de Estado da Agricultura Prefeitura Municipal de Nova Prata, p.265-278.

Krügel, M. M. \& Behr, E. R. 1999. Consumo dos frutos de Alchornea triplinervia (Euphorbiaceae) por aves em fragmentos florestais urbanos de Maringá, Paraná. Biotemas 12(1):149-155.

LECK, C. F. 1972. The impact of some North American migrants at fruiting trees in Panamá. Auk 89:842-850.

Leite, P. F. \&. Klein, R. M. 1990. Vegetação. In: Mesquita, O. V. coord. Geografia do Brasil - Região Sul. Rio de Janeiro, IBGE. p.113-150.

LEVEY, D. J. 1987. Seed size and fruiting handling techniques of avian frugivores. American Naturalist 129(4):471-485.

Loiselle, B. A. \& Blake, J. G. 1999. Dispersal of melastome seeds by fruit-eating birds of tropical forest understory. Ecology 80(1):330-336

Lorenzi, H. 1992. Árvores brasileiras: manual de identificação e cultivo de plantas arbóreas nativas do Brasil. São Paulo, Plantarum. 352p.

Machado, P. F. S. \& Longhi, S. J. 1990. Aspectos florísticos e fitossociológicos do "Morro do Elefante", Santa Maria, RS. Revista Centro de Ciências Rurais 20(3-4):261-280.

MARChIORI, J. N. C. 1997 Dendrologia das angiospermas: das magnoliáceas às flacourtiáceas. Santa Maria, UFSM. 271p.

Marini, M. Â. \& Cavalcanti, R. B. 1998. Frugivory by Elaenia flycatchers. Hornero 15:47-50.

McDiarmid, R.W.; Ricklefs, R. E. \& Foster, M. 1977. Dispersal of Stemmadenia donnell-smithii (Apocynaceae) by birds. Biotropica 9(1):9-25.

MCKEY, D. 1975. The ecology of coevolved seed dispersal systems. In: Gilbert, L. E. \& Raven, P. H. eds. Coevolution of animals and plants. Austin, University of Texas Press. p.159-191.

Mikich, S. B. 2002. A dieta frugívora de Penelope superciliaris 
(Cracidae) em remanescentes de floresta estacional semidecidual no centro-oeste do Paraná, Brasil e sua relação com Euterpe edulis (Arecaceae). Ararajuba 10(2):207-217.

Moermond, T. C. \& Denslow, J. S. 1985. Neotropical avian frugivores: patterns of behavior, morphology, and nutrition, with consequences for fruit selection. In: Buckley, P. A., Foster, M. S., Morton, E. S., Ridgely, R. S. \& Buckley, F. G. eds. Neotropical ornithology. Lawrence, AOU (Ornithological Monographs, 36). p.865-897.

Pereira, P. R. B.; Netto, L. R. G.; Borin, C. J. A \& Sartori, M. G. B. 1989. Contribuição a geografia física do município de Santa Maria: unidades de paisagem. Geografia: Ensino \& Pesquisa 3:37-68.

Pineschi, R. B. 1990. Aves como dispersores de sete espécies de Rapanea (Myrsinaceae) no maciço do Itatiaia, Estados do Rio de Janeiro e Minas Gerais. Ararajuba 1:73-78.

Pizo, M. A. 1996. Frugivoria e dispersão de sementes por aves. In: Congresso Brasileiro de Ornitologia, $5^{\circ}$, Campinas. Anais... Campinas, UNICAMP. p.163-170.

1997. Seed dispersal and predation in two populations of Cabralea canjerana (Meliaceae) in the Atlantic Forest of southeastern Brazil. Journal of Tropical Ecology 13:559578.

Pizo, M. A.; Silva, W. R.; Galetti, M. \& Laps, R. 2002. Frugivory in cotingas of the Atlantic Forest of southeast Brazil. Ararajuba 10(2): 177-185.

Reitz, R.; Klein, R. M. \& Reis, A. 1988. Projeto Madeira do Rio Grande do Sul. Porto Alegre, SUDESUL, DRNR, Herbário Barbosa Rodrigues. 525p.

Rohwer, J. G. \& KUBITZKi, K. 1993. Ecogeographical differentiation in Nectandra (Lauraceae), and its historical implications. Botanica Acta 106:88-99.

Santana, E. \& Milligan, B. G. 1984. Behavior of toucanets, bellbirds, and quetzals feeding on Lauraceous fruits. Biotropica 16(2): 152-154.

SAS (Statistical Analysis System) 1989. User's Guide. Version 6. 4 ed. North Caroline, SAS Institute Inc. $846 \mathrm{p}$.

Schupp. E. W. 1993. Quantity, quality and the effectiveness of seed dispersal. Vegetatio 107/108:15-29.

SICK, H. 1997. Ornitologia brasileira. Rio de Janeiro, Nova Fronteira. $912 \mathrm{p}$.

Skutch, A. F. 1944. Life history of the quetzal. Condor 46:213-235

SNOw, B. K. 1970. A field study of the bearded bellbird in Trinidad. Ibis 112: 299-329.

Snow, D. W. 1981. Tropical frugivorous birds and their food plants: a world survey. Biotropica 13(1):1-14.

Solórzano, S.; Castillo, S.; Valverde, T. \& Ávila, L. 2000. Quetzal abundance in relation to fruit availability in a cloud forest in Southeastern México. Biotropica 32(3):523-532.

WheElwright, N. T. 1983. Fruits and the ecology of Resplendent Quetzals. Auk 100:286-301. 1985. Fruit size, gape width, and the diets of fruit-eating birds. Ecology 66:808-818.

Wheelwright, N. T.; Haber, W. A.; Murray, K. G. \& Guindon, C. 1984. Tropical fruit-eating birds and their food plants: a survey of a Costa Rican lower montane forest. Biotropica 16(3):173-192.

Willis, E. O. 1966. Competitive exclusion and birds at fruiting trees in Western Colombia. Auk 83:479-480.

Recebido em junho de 2004. Aceito em novembro de 2005. ISSN 0073-4721

Artigo disponível em: www.scielo.br/isz 